\title{
Attitudes toward Genetic Research Review: Results from a Survey of Human Genetics Researchers
}

\author{
K.L. Edwards ${ }^{a} \quad$ A.A. Lemke ${ }^{c}$ S.B. Trinidad ${ }^{b}$ S.M. Lewis ${ }^{d} \quad H$. Starks $^{b}$ \\ M.T. Quinn Griffin ${ }^{\mathrm{e}}$ G.L. Wiesner ${ }^{f}$ The GRRIP Consortium \\ Departments of a Epidemiology and ${ }^{\mathrm{b}}$ Bioethics and Humanities, University of Washington, Seattle, Wash., \\ ${ }^{c}$ Center for Genetic Medicine, Northwestern University, Chicago, Ill., ${ }^{d}$ Center for Genetic Research Ethics and Law, \\ e Frances Payne Bolton School of Nursing, and ${ }^{\mathrm{f}}$ Department of Genetics and Medicine, Case Western \\ Reserve University, Cleveland, Ohio, USA
}

\section{Key Words}

Genomics • Human genetics • Institutional Review Board • Survey

\begin{abstract}
Background: Researchers often relate personal experiences of difficulties and challenges with Institutional Review Board (IRB) review of their human genetic research protocols. However, there have been no studies that document the range and frequency of these concerns among researchers conducting human genetic/genomic studies. Methods: An online anonymous survey was used to collect information from human genetic researchers regarding views about IRB review of genetic protocols. Logistic regression was used to test specific hypotheses. Results from the national online survey of 351 human genomic researchers are summarized in this report. Results: Issues involving considerable discussion with IRBs included reconsent of subjects (51\%), protection of participants' personal information (39\%) and return of results to participants (34\%). Over half of the participants had experienced one or more negative consequences of the IRB review process and approximately $25 \%$ had experienced
\end{abstract}

one or more positive consequences. Respondents who had served on an IRB were about $80 \%$ more likely to report positive consequences of IRB review than their colleagues who had never served on an IRB $(p=0.03)$. Survey responses were mixed on the need for reconsent before data sharing and risks related to participant reidentification from genomic data. Conclusion: The results from this study provide important perspectives of researchers regarding genetic research review and show lack of consensus on key research ethics issues in genomic research.

Copyright $\odot 2011$ S. Karger AG, Basel

The expansion of genome sciences has led to an improved understanding of the genetic component of human disease and the development of new medical treatments. Clearly, these accomplishments could not have been possible without the participation of individuals and their families in genetic research protocols vetted and approved by Institutional Review Boards (IRBs).

US federally-funded research using human participants requires IRB review and oversight prior to implementation with at least an annual review for each year of

\section{KARGER}

(c) 2011 S. Karger AG, Basel

Fax +41613061234 E-Mail karger@karger.ch www.karger.com www.karger.com/phg
Karen L. Edwards, PhD

University of Washington, Department of Epidemiology, Box 354921

6200 NE 74th St., Bldg. 29, Suite 250

Seattle, WA 98115 (USA)

Tel. +1 206616 1258, E-Mail keddy@uw.edu 
the project [1-5]. The first step in this process is often to determine whether the project involves human subjects and requires IRB review. This can be problematic in multi-center studies conducted in the US, as local guidance can vary on what qualifies as human subjects research. For example, in some states, deceased subjects are considered human subjects, and research use of their information requires IRB approval. Similarly, local differences exist with regard to whether use of coded data with no links to personal identifiers qualifies as human subjects research. In informal and professional settings, genetic researchers often relate personal experiences of difficulties and challenges with the IRB review process, particularly in the context of rapid technological advances, multi-institutional studies and the generation of dense genomic data for large groups of participants. Anecdotal reports suggest that the review process for genetic studies is more complicated than for nongenetic studies in that genetic studies take longer to review and, as is seen with other interinstitutional research, the same protocol can generate varying opinions from local IRBs.

Additional challenges for genetic researchers are seen with the emergence of large-scale genomic studies which require protections not only for the initial studies, but for future, unforeseen use of the primary genetic samples and/or data. Some have argued that the standard processes of informed consent and de-identification of study data may be less effective for these types of studies [6-10]. Thus, the regulatory framework for informed consent and risk-benefit assessment for data sharing in genetic and genomic studies may be an additional source of disagreement between researchers and their IRBs.

Although previous studies have documented some of the tensions around the conduct of human genetic research $[9,11,12]$, it is not known how often these problems are encountered by genetic researchers, how widespread they may be and what, if any, interventions or policies should be developed to address these issues. Further, studies to collect comparable data from IRB professionals are needed to identify areas of common concern and disagreement between these 2 groups. The Genetics Research Review and Issues Project (GRRIP) was designed to address this gap by conducting parallel surveys of both genomics researchers and IRB staff, administrators and members.

We report the results from a national survey of human genetic researchers here. An article summarizing the results from a parallel survey of IRB professionals is published elsewhere [13], and a third paper comparing the responses from both groups is in process.

\section{Methods}

Overview of Genetics Research Review and Issues Project

GRRIP is a collaborative project between geneticists, health researchers, bioethicists, and representatives of community and professional societies with an overall goal of documenting the current issues related to the regulation of genetic and genomic research and developing workable solutions to improve the conduct of future research.

The GRRIP consists of 3 phases: Phases 1 and 2 were focused on qualitative and quantitative data collection from human genetic researchers and IRB staff, administrators and members; and Phase 3 focuses on data analysis and dissemination. Protocols and recruitment methods for Phases 1 and 2 were developed in collaboration with the American Society of Human Genetics (ASHG) and Public Responsibility in Medicine and Research (PRIM\&R) representatives and approved by the Executive Board of each organization. All protocols for this project were reviewed and approved by the IRB from the lead institution for the overall GRRIP consortium (University of Washington) as well as by the other institutions participating in the GRRIP.

\section{Development of the Survey}

The purpose of the survey was to document the range and frequency of occurrence of issues related to IRB review of human genetic protocols. Data from a convenience sample of 25 researchers participating in qualitative interviews and focus groups were utilized to identify the major content areas and to inform potential response categories for the survey. The survey was then developed using the Tailored Design Method [14] as a general guide.

The resulting instrument had a total of 113 questions, including a subset of demographic questions. The survey was divided into 5 general topic areas: the IRB application process, the IRB review process, IRB functions, design-specific issues in genetic research, and background information of the participants. The types of response categories varied based on the question and included either 'yes/no/not sure' options, Likert scales (e.g. 5-point scales rating agreement, likelihood or importance of the statement with a sixth 'don't know' or 'it depends' option) or categorical responses.

All survey questions were pretested among members of the GRRIP consortium and with independent reviewers. Cognitive interviews were also conducted with 9 genetics researchers, and changes were made to improve the clarity of the survey questions [15]. Pilot testing of the web-based survey was conducted to assess any technical difficulties and to confirm the length of time required to complete the final instrument.

The Catalyst WebQ survey software, developed by the University of Washington, was used to develop and implement the survey. The survey was administered anonymously and participants were free to skip any questions that they did not wish to answer. The instrument is available as an online supplement.

Recruitment and Eligibility of Genetic Researchers

The sample was recruited from the current electronic membership list of the ASHG. Members were invited to participate in an anonymous, web-based survey via introductory email sent from the executive vice president of the organization. The first email invitation was sent to all current members $(n=4,908)$ of 
ASHG on April 28, 2009, including a link to the survey. A second email was sent to the same list 2 weeks later to thank individuals for their participation and to remind them to complete the online survey if they had not already done so. In the second email, members also were asked to forward the invitation and survey link to other, non-ASHG members of their professional community. The first question on the survey assessed eligibility by asking whether participants conducted human genetics research; if they did not, a skip pattern was programmed to collect only background information for comparison with other survey respondents.

\section{Statistical Analysis}

Responses to all questions were first summarized using frequency distributions. Response categories for some of the questions were collapsed into fewer categories for testing and to facilitate interpretation. For example, the 5 categories of the Likert scales were collapsed into 3, combining the 'strongly' and 'somewhat' categories (e.g. strongly agree, somewhat agree and strongly disagree, somewhat disagree).

Specific questions were then selected for further analyses and statistical testing. Two hypotheses were generated from Phase 1 key informant interview data: (1) researchers who had served on an IRB might differ in their experiences with the IRB from those who had never served, and (2) clinical investigators may report different experiences with the IRB compared to their nonclinical colleagues. To address these 2 hypotheses, data from questions asking about service on an IRB and the activity (or appointment type) that occupies more than $50 \%$ of their time (research, teaching, administration, clinical, counseling, other) were used. Responses to the question on type of activity were used to classify respondents as either primarily clinical (medical or counseling) or research-focused investigators. Those answering teaching, administration or other as their primary activity were excluded from analyses intended to compare clinicians and nonclinicians.

As respondents could list multiple consequences of IRB review, ordinal logistic regression was used to assess the relationship between service on an IRB (ever/never) or type of activity (clinical vs. research) and consequences of IRB review, including adjustment for sex and years of research experience ( $\leq 5$ years, $6-15$ years, $>15$ years). The outcome, degree of negativity/positivity, was defined by counting the number of negative and positive consequences to the single question asking about consequences of IRB review and ordering them as follows: 3-7 negative comments, 1-2 negative comments, more negative than positive comments, equal numbers of negative and positive comments, more positive than negative comments, and all positive comments.

Odds ratios and $95 \%$ confidence intervals were estimated for each covariate included in the models. Stata version 9.2 was used for all analyses [16]. A p $\leq 0.05$ was considered statistically significant for all tests. Sample sizes varied by question because participants were allowed to skip any question they did not wish to answer, and some questions had built-in skip patterns. Finally, eligible respondents from outside the US were included in the analysis as their responses were not substantially different from the US-based respondents.

\section{Results}

A total of 372 individuals completed the online survey; 266 individuals responded within 2 weeks of receiving the first letter of invitation and 106 following the second request. Of the 372 respondents, 21 had never conducted human genetics research and were excluded from all analyses. Demographic characteristics were similar for ineligible and eligible respondents (data not shown). Table 1 presents the demographic information for the 351 eligible respondents. Slightly more women $(53 \%)$ than men responded to the survey. The majority of respondents were US-based and had been conducting genetic research for 10 or more years. The majority of non-USbased respondents were from Canada $(n=16)$, followed by the UK $(n=6)$, Australia ( $=5)$, Italy $(n=4)$, and Germany and Japan $(n=3$ each), with responses from a total of 21 countries. More than half of all respondents spend more than $50 \%$ of their time conducting research, followed by $23 \%$ in clinical service. Over a quarter of the respondents had served on an IRB, with $37 \%$ of those serving for more than 5 years and $4 \%$ serving for less than 1 year. The majority of the respondents were members of the ASHG (99\%), including $97 \%$ of the non-US-based respondents, and about $2 \%$ also were members of PRIM\&R, including 3.3\% of the non-US-based respondents.

\section{IRB Application Process}

In terms of views toward the IRB application process, $64 \%$ of the respondents agreed that their IRB provides useful guidance in preparing a new IRB application for genetic studies, while $21 \%$ disagreed with this statement. Types of guidance provided by the IRB for preparing new applications include online web-based (55\%) or hardcopy instructions (33\%), in-person meetings (34\%) and phone (45\%) or email consultation (47\%). Nearly $50 \%$ of the respondents indicated that all of these types of guidance were available to them, but 3\% indicated that none of these were available. Nearly all respondents (93\%) felt that guidance from their IRB in writing a consent form was very or somewhat important, followed by help with planning for sharing genetic research data with other investigators (75\%). In contrast, only $29 \%$ felt that guidance from the IRB in developing a genetic research study design was important.

\section{IRB Review Process}

Respondents were asked whether they generally agree with their IRB about whether a given genetics project requires IRB review: 78\% agreed that they did. The next 
Table 1. Characteristics of eligible human genetic researchers participating in the survey

\begin{tabular}{|c|c|c|c|}
\hline Question & Response & Frequency & Percentage \\
\hline \multirow[t]{2}{*}{ Gender } & Female & 182 & 53.1 \\
\hline & Male & 161 & 46.9 \\
\hline \multirow[t]{4}{*}{ Country of primary affiliation } & US & 287 & 82.7 \\
\hline & Canada & 16 & 4.6 \\
\hline & Other & 44 & 12.7 \\
\hline & Total responses & 347 & 98.9 \\
\hline \multirow[t]{5}{*}{ Number of years conducting human genetic research } & $<2$ years & 7 & 2.0 \\
\hline & $2-5$ years & 54 & 15.4 \\
\hline & $6-10$ years & 56 & 16.0 \\
\hline & $11-15$ years & 66 & 18.9 \\
\hline & $>15$ years & 167 & 47.7 \\
\hline \multirow[t]{2}{*}{ Served on an IRB } & Yes & 94 & 27.1 \\
\hline & No & 253 & 72.9 \\
\hline \multirow[t]{5}{*}{ Academic rank } & Professor & 114 & 32.9 \\
\hline & Associate Professor & 73 & 20.0 \\
\hline & Assistant Professor & 75 & 21.6 \\
\hline & Instructor & 12 & 3.5 \\
\hline & Not applicable & 73 & 21.0 \\
\hline \multirow[t]{7}{*}{ Most frequent work activity } & Research & 213 & 62.1 \\
\hline & Clinical & 78 & 22.7 \\
\hline & Administration & 23 & 6.7 \\
\hline & Teaching & 11 & 3.21 \\
\hline & Counseling & 9 & 2.6 \\
\hline & Other & 9 & 2.6 \\
\hline & Total responses & 343 & 97.7 \\
\hline
\end{tabular}

Table 2. Consequences of the IRB review process $(\mathrm{n}=341)$

Question: In your experience, which of the following consequences (if any) have occurred as a result of the IRB review process of a genetic study?

\begin{tabular}{lrr}
\hline Consequence & Frequency & Percentage \\
\hline Negative consequences & 184 & 54.0 \\
Excessive delay of a project & 79 & 27.9 \\
Dissuaded me or my colleagues from pursuing similar projects in the future & 74 & 23.2 \\
Put me at a competitive disadvantage with peers & 54 & 21.7 \\
Threatened collaborations or relations with research partners & 21 & 15.8 \\
Required that I make substantive changes to my study design & 19 & 6.2 \\
Lost funding for a project & 5.6 \\
Affected my (or my institution's) ability to recruit new investigators & 86 & 77 \\
Positive consequences & 41 & 25.2 \\
Improved participant protections & 22.6 \\
Helped me plan for future research (e.g. broadened consent language) & 62 \\
Identified potential harms to subjects that were not addressed & 3 \\
None of the above & 12.0 \\
All of the above & 18.2 \\
Other & 0.9 \\
\hline
\end{tabular}


Table 3. Issues causing considerable discussion $(n=338)$

Question: Which of the following issues (if any) have required considerable discussion between you and your IRB in the review of genetic research applications? By 'considerable discussion' we mean more than 2 or 3 back-and-forth rounds and/or more than a 1-h conversation. Please select all that apply.

\begin{tabular}{lcc}
\hline Issue & $\mathrm{n}$ & Percentage \\
\hline Informed consent process or documentation & 172 & 50.9 \\
Procedures for protecting participants' personal information or samples & 132 & 39.1 \\
Return of genetic research results to participants & 115 & 34.0 \\
Reconsent from research participants for a new study or change in purpose & 101 & 29.9 \\
Study intent & 60 & 17.8 \\
Plans, or lack of plans, to deal with community harms and benefits & 37 & 11.0 \\
\hline None of the above & 92 & 27.2 \\
All of the above & 20 & 5.9 \\
Other & 21 & 6.2 \\
\hline
\end{tabular}

questions asked about different consequences of the IRB review process and the frequency of their occurrence (table 2). The most common consequence was excessive delay of a project as a result of the IRB review process. We also analyzed these individual consequences grouped as either having a positive or negative effect on research and found that over half the respondents reported one or more negative consequences. About 25\% of the survey respondents had experienced one or more positive consequences, while nearly $20 \%$ had not experienced any of the listed consequences.

The data were then analyzed using ordinal logistic regression analysis to evaluate the effect of ever serving on an IRB and primary type of activity (clinical vs. research). Researchers who had served on an IRB were significantly $(p=0.03)$ more likely to report a higher degree of positivity compared to those who had never served on an IRB $(\mathrm{OR}=1.79 ; 95 \% \mathrm{CI}=1.05-3.05)$, including adjustment for gender and years of research experience. Respondents who were primarily engaged in clinical activities were $35 \%$ more likely to report a higher degree of negativity $(\mathrm{OR}=0.65 ; 95 \% \mathrm{CI}=0.41-1.05)$ compared to their colleagues involved primarily in research activities, which was of borderline statistical significance $(\mathrm{p}=0.08)$. In the full ordinal logistic regression model including both these predictors and adjusting for gender and years of research experience, having ever served on the IRB was no longer statistically significant $(\mathrm{OR}=1.56$; $95 \% \mathrm{CI}=0.87$ $2.79 ; \mathrm{p}=0.14)$, but type of activity remained borderline significant $(\mathrm{OR}=0.64 ; 95 \% \mathrm{CI}=0.40-1.03 ; \mathrm{p}=0.07)$.

We also sought to understand the types of issues in the review process that cause 'considerable' discussion with their IRB for genetic research applications. Issues related to informed consent were the most frequent cause for discussion, followed by return of research results and reconsent (table 3). Twenty-seven percent of the respondents had not experienced any of the issues listed.

\section{IRB Functions}

We asked questions about whether specific functions and roles of the IRB, such as assessing scientific rigor of studies, assessing the risks of research, protecting the institution from potential liability, and determining appropriate safeguards against those risks, and whether they should be considered differently for genetic research compared to other types of human research. For all of these issues, the majority of respondents $(75-88 \%)$ indicated that there should be no difference in how research is reviewed.

\section{Specific Genetic Research Issues}

In 2007, the National Institutes of Health (NIH) implemented a policy for sharing data obtained in NIH-supported or -conducted genome-wide association studies (GWAS) (http://grants.nih.gov/grants/gwas/). The goal of the policy is to advance science for the benefit of the public through providing broad and consistent access to GWAS datasets. We asked respondents to indicate their level of agreement with the following statement: 'The $\mathrm{NIH}$ guidelines for sharing data from genome-wide association studies (GWAS) are clear.' Forty-three percent agreed that the guidelines are clear, 12\% disagreed with the statement and 36\% indicated that they did not know whether the guidelines are clear; $9 \%$ expressed a neutral 
Table 4. Views toward return of results

\begin{tabular}{|c|c|c|c|c|c|c|c|}
\hline Question (sample size) & $\begin{array}{l}\text { Strongly } \\
\text { agree }\end{array}$ & $\begin{array}{l}\text { Somewhat } \\
\text { agree }\end{array}$ & $\begin{array}{l}\text { Neither agree } \\
\text { nor disagree }\end{array}$ & $\begin{array}{l}\text { Somewhat } \\
\text { disagree }\end{array}$ & $\begin{array}{l}\text { Strongly } \\
\text { disagree }\end{array}$ & $\begin{array}{l}\text { Don't } \\
\text { know }\end{array}$ & $\begin{array}{l}\text { It } \\
\text { depends }\end{array}$ \\
\hline \multicolumn{8}{|c|}{$\begin{array}{l}\text { Researchers have an ethical obligation to return individual research results (genetic or non-genetic): } \\
\text { That would affect a participant's health or health care } \\
\text { (assume procedures were performed in a CLIA-certified }\end{array}$} \\
\hline laboratory) $(\mathrm{n}=347)$ & $52.7 \%$ & $29.7 \%$ & $8.0 \%$ & $3.8 \%$ & $3.8 \%$ & $2.0 \%$ & NA \\
\hline If a participant asks for them $(n=347)$ & $30.8 \%$ & $18.7 \%$ & $6.1 \%$ & $15.0 \%$ & $14.1 \%$ & $0.6 \%$ & $14.7 \%$ \\
\hline $\begin{array}{l}\text { If the investigator feels that the participant might be interested } \\
\text { in knowing about them }(\mathrm{n}=350)\end{array}$ & $8.6 \%$ & $16.3 \%$ & $13.4 \%$ & $18.9 \%$ & $32.0 \%$ & $1.1 \%$ & $9.7 \%$ \\
\hline $\begin{array}{l}\text { Ethical obligations to return results are the same for investigators } \\
\text { who enrolled participants in a study and for investigators who } \\
\text { utilized coded data from a shared repository }(\mathrm{n}=348)\end{array}$ & $16.1 \%$ & $15.8 \%$ & $8.6 \%$ & $25.3 \%$ & $28.5 \%$ & $5.8 \%$ & NA \\
\hline $\begin{array}{l}\text { For large-scale studies, the logistical challenges involved in } \\
\text { returning individual research results to participants (e.g. } \\
\text { additional expense, anticipated difficulty in contacting } \\
\text { participants) makes this practically impossible for most } \\
\text { researchers }(\mathrm{n}=348)\end{array}$ & $31.3 \%$ & $34.8 \%$ & $9.2 \%$ & $17.2 \%$ & $3.7 \%$ & $3.7 \%$ & NA \\
\hline $\begin{array}{l}\text { Research on coded data from a repository may sometimes yield } \\
\text { clinically significant results. A system should be in place that } \\
\text { allows users of the coded data to report such results to the } \\
\text { repository, so that decisions about return of results can be made } \\
\text { by the original, submitting investigator with the help of } \\
\text { additional experts and the IRB }(\mathrm{n}=348)\end{array}$ & $31.6 \%$ & $41.1 \%$ & $8.3 \%$ & $8.1 \%$ & $6.6 \%$ & $4.3 \%$ & NA \\
\hline
\end{tabular}

opinion. Most respondents (57\%) agreed that genetic research using coded human tissue specimens (when the researchers do not have access to the link between identifiers and samples) constitutes human subjects research, and therefore requires IRB review.

Respondents were also asked about their views on both the risk of participant reidentification and the likelihood of such reidentification resulting in harm to participants. Seventy percent of respondents believed that the reidentification of research participants in a study involving coded genetic data was unlikely to occur. If a study participant were to be reidentified, $76 \%$ of respondents thought it was unlikely that the participant would be harmed as a result. With respect to the possibility of an investigator being compelled to disclose information about genetic research participants (e.g. by a law-enforcement agency), about half of the respondents considered this unlikely, while a quarter considered it likely, and 18\% said they did not know.

With respect to views on returning research findings (table 4), the majority (82\%) of researchers felt that - assuming testing was performed in a laboratory that had received appropriate certification for clinical testing under the federal Clinical Laboratory Improvement Amendments (CLIA) (http://www.cms.hhs.gov/clia/) - there is an ethical obligation to return research results (genetic or nongenetic) that would affect a participant's health or health care. However, $31 \%$ of respondents indicated that their response would change if tests were not conducted in a CLIA-certified laboratory. About half the participants agreed that results should be returned if the participant asked for them, while only $25 \%$ indicated that results should be returned if the investigator felt that the participant might be interested in knowing about them. Two-thirds of the respondents indicated that the logistics involved in returning results to participants would make it practically impossible to implement in large-scale studies.

Respondents reported a greater diversity of opinion about when it is ethically necessary to obtain reconsent from research participants. We asked about a number of different scenarios, including protocol changes, changes in study purpose, data-sharing, and pediatric subjects who have increased decisional capacity since the time of enrollment. Respondents generally agreed that reconsent would be required in 3 of the scenarios: if the investigator wants to investigate an unrelated condition or clinical manifestation (75\%), if the investigator wants to add genetic measures to a study that did not originally include them $(66 \%)$ or if the original consent was given by a mi- 
nor subject's parents and the subject is now old enough to decide for him or herself (57\%). The majority (74\%) of respondents also noted that the logistics involved in obtaining reconsent would make it practically impossible for most researchers to implement.

Views were split on the 3 other reconsent scenarios. The first was if the researcher wants to look for a genetic variant or gene other than the one mentioned in the original consent form ( $52 \%$ agreed vs. $40 \%$ disagreed). The second was if the researcher wants to investigate a different, but related, condition or clinical manifestation (45\% agreed and $46 \%$ disagreed). The third was whether reconsent was necessary when sharing de-identified samples or data (without a linkage file) with an investigator at another institution (43\% agreed while $44 \%$ disagreed).

\section{Discussion}

This study offers a snapshot of researchers' views about IRB review and participant protections in human genetic research. Overall, respondents reported fewer issues with the overall IRB application process compared with the IRB review process. Most respondents believed that IRBs should review genetic research applications the same way they review other biomedical research applications. These results suggest that, in the ongoing debate about genetic exceptionalism [17-19], most investigators working in the field may consider genetic information to be one more kind of health information, deserving of the same level of protection as other such data.

Some of our findings are consistent with the anecdotal accounts from colleagues that provided the impetus for this project. While some respondents reported that IRB review has had positive effects on their work (including helping investigators plan for future research), many more pointed to negative effects. Of note, more than half reported that review had resulted in excessive delay of a project, and 35\% agreed that IRB review takes longer for projects with a genetic component. Although our results report respondents' perceptions, rather than actual comparative data on review duration for genetic vs. nongenetic applications, timeliness of review appears to be a significant concern for respondents. About 20\% reported that lengthy review had threatened collaborations or relations with research partners, which is consistent with published accounts of difficulties associated with managing participant protections in large multi-center consortia [20-23].

Genetic Research Review
In adjusted analyses, respondents who had served on an IRB reported significantly fewer negative consequences of IRB review than their colleagues who had not served on an IRB, possibly reflecting a greater familiarity with the IRB review process. Occupational focus (clinical vs. research) was only of borderline significance. These results, particularly those relating to prior service on an IRB, highlight potential opportunities for more focused work to identify the reason for these differences.

Our findings demonstrate substantial variation in respondents' views about when reconsent is ethically necessary. Although there was strong agreement that reconsent is appropriate when the proposed use is clearly outside the bounds of the original consent language (i.e. to investigate an unrelated condition or to add genetic measures to a study that did not include them), opinions were almost evenly divided about cases in which the new use could be construed to be within the spirit of the original consent, such as when the researcher wants to investigate a different but related condition, to investigate a different gene or genetic variant or to share participants' de-identified sample or data with an investigator at another institution. Whether these differences in opinion reflect respondents' personal convictions, variations in local practice and IRB policy, or both, these results highlight an important area for further investigation, education and consensus development among genetic researchers.

Questions about the need to reconsent also depend on the wording of the original consent form, as indicated by several respondents to the online survey. Ninety-three percent of researchers participating in this survey indicated that guidance from their IRB in writing a consent form for genetic studies is important, while only $1.5 \%$ indicated that this is not the role of the IRB. When asked about the use of a broad consent form that anticipates future genetic research studies (e.g. such as establishing a biobank and/or studying additional diseases), about half the respondents indicated that this practice is acceptable to their IRB. General agreement about the appropriate scope and content of informed consent for genomic research may help to reduce tensions between researchers and their IRBs, as the consent form can impact a number of areas identified in this survey as requiring considerable discussion with their IRB. A number of groups have proposed language for model consent forms [24, 25], but it is not known to what extent researchers use these resources. Perhaps further exploration and discussion of the values that researchers and their IRBs hold regarding reconsent in various scenarios can take place within the

Public Health Genomics 2011;14:337-345 
appropriate professional organizations and in local and national discussions among stakeholders. Finally, approaches to reduce some of the administrative burdens associated with preparing an application for IRB review should also be considered.

Nearly all investigators (99\%) agreed that the role and functions of the IRB should include protection of human subjects. However, it is interesting to note that about half the respondents felt that harms resulting from genetic studies are very unlikely, particularly in the possible identification of subjects from coded data and whether the subject would be harmed if identified (49 and 53\%, respectively).

Finally, the survey results presented here indicate that some respondents believe that their or their institution's ability to recruit new investigators was negatively affected by the IRB review process. Although the numbers were small, these findings suggest that communication between researchers and their IRBs can be improved at some institutions. Finding solutions that will facilitate genetic research, while at the same time ensuring protection of human subjects, will be essential.

There are several limitations of the study, including the generalizability of these findings to the broader genetic research community. While the response rate is estimated to be $7.5 \%$ (372 out of 4,908), it is difficult to obtain a precise estimate of the denominator for 2 reasons: first, not all members of ASHG are eligible to participate in the study as they do not conduct human genetics research, and secondly, it is not known how many ASHG members forwarded the survey to colleagues. Further, among those that did respond, it is possible, even likely, that those people choosing to respond to the survey were different in terms of their experience with IRBs than the nonrespondents. In spite of these limitations, the information provided by this survey is important in documenting the range and frequency of concerns among this group of researchers as well as the impact and implications of IRB review on genetic research.

In conclusion, the results from this study document the perspectives of researchers in human genetics and point to several opportunities for improvement in the human subjects review of genetic studies. In particular, we have identified specific areas where there is a lack of agreement among researchers or where guidance is needed. The findings from this study offer a useful starting point for human genetic researchers and their professional organizations to further discuss and address these often challenging and controversial issues. Engaging key stakeholders in this process can serve as a catalyst for changing guidelines within organizations with the goal of informing a set of policies around these issues.

\section{Acknowledgements}

Support for this research comes from National Human Genome Research Institute grants: P50 HG003374; P50 HG003390; U01 HG004609. This work was presented in part at the American Society for Human Genetics Annual Meeting in Honolulu, Hawaii, in October 2009, and at the Public Responsibility in Medicine and Research Advancing Ethical Research Conference in Nashville, Tennessee, in November 2009.

This investigation involved multiple collaborators including the University of Washington Center for Genomics and Healthcare Equality, Case Western Reserve University Center for Genetic Research Ethics and Law, Public Responsibility in Medicine and Research (PRIM\&R), the American Society of Human Genetics (ASHG), and the Genetics Research Review and Issues Project (GRRIP) investigators. Additional GRRIP project investigators include (in alphabetical order): Joann Boughman, Wylie Burke, Lynn Dressler, William Freeman, Nancy Gerson, Eric Juengst, Patricia Marshall, P. Pearl O'Rourke, Roselle Ponsaran, and Nancy Press. The authors also wish to thank Ms. Jia Yin Wan and Ms. Katherine Snapinn for their assistance in organizing and analyzing the data and in preparing the manuscript for publication.

\section{References}

1 Burke GS: Looking into the institutional review board: observations from both sides of the table. J Nutr 2005;135:921-924.

2 Burris S, Moss K: U. S. Health researchers review their ethics review boards: a qualitative study. J Empir Res Hum Res Ethics 2006; 1:39-58.

3 Code of Federal Regulations (CFR): Title 45, part 46, protection of human subjects, DHHS. 2005. http://ohsr.od.nih.gov/guidelines $/ 45 \mathrm{cfr} 46 . \mathrm{html}$
4 Lynn MR, Nelson DK: Common (mis)perceptions about IRB review of human subjects research. Nurs Sci Q 2005;18:264-270.

5 Whitney SN, Alcser K, Schneider C, McCullough LB, McGuire AL, Volk RJ: Principal investigator views of the IRB system. Int J Med Sci 2008;5:68-72.

6 Annas GJ: Commentary - reforming informed consent to genetic research. JAMA 2001;286:2326.
7 Ellerin BE, Schneider RJ, Stern A, Toniolo PG, Formenti SC: Ethical, legal, and social issues related to genomics and cancer research: the impending crisis. J Am Coll Radiol 2005;2:919-926.

8 Hunter AG: Is multicenter collaborative research in clinical genetics dead and, if so, what killed it? Am J Med Genet A 2005;134: 237-239.

-9 McGuire AL, Gibbs RA: Genetics. No longer de-identified. Science 2006;312:370-371. 
10 O’Neill O: Informed consent and genetic information. Stud Hist Philos Sci C Stud Hist Philos Biol Biomed Sci 2001;4:689-704.

11 Caulfield T, Upshur RE, Daar A: DNA databanks and consent: a suggested policy option involving an authorization model. BMC Med Ethics 2003;4:E1.

12 McGuire AL, Gibbs RA: Meeting the growing demands of genetic research. J Law Med Ethics 2006;34:809-812.

-13 Lemke AA, Edwards KL, Trinidad SB, Starks H, Wiesner GL; GRRIP Consortium: Attitudes toward genetic research review: results from a national survey of institutional review board members, administrators, and staff. J Empir Res Hum Res Ethics 2010;5: 83-91.

14 Dillman DA: Mail and Internet Surveys: The Tailored Design Method. Hoboken, Wiley, 2007.

15 Willis GB: Cognitive Interviewing: A Tool for Improving Questionnaire Design. Thousand Oaks, Sage Publications, 2005.
16 StataCorp: Stata statistical software: Release 9.2, 2001. College Station, TX, Stata Corporation.

17 Evans JP, Burke W: Genetic exceptionalism. Too much of a good thing? Genet Med 2008; 10:500-501.

18 Kakuk P: Gene concepts and genethics: beyond exceptionalism. Sci Eng Ethics 2008; 14 357-375.

19 McGuire AL, Fisher R, Cusenza P, Hudson K, Rothstein MA, McGraw D, Matteson S, Glaser J, Henley DE: Confidentiality, priva$\mathrm{cy}$, and security of genetic and genomic test information in electronic health records: points to consider. Genet Med 2008;10:495499.

20 Greene SM, Geiger AM: A review finds that multicenter studies face substantial challenges but strategies exist to achieve Institutional Review Board approval. J Clin Epidemiol 2006;59:784-790.

21 Greene SM, Geiger AM, Harris EL, Altschuler A, Nekhlyudov L, Barton MB, Rolnick SJ, Elmore JG, Fletcher S: Impact of IRB requirements on a multicenter survey of prophylactic mastectomy outcomes. Ann Epidemiol 2006;16:275-278.
22 McWilliams R, Hoover-Fong J, Hamosh A, Beck S, Beaty T, Cutting G: Problematic variation in local institutional review of a multicenter genetic epidemiology study. JAMA 2003;290:360-366.

23 Sherwood ML, Buchinsky FJ, Quigley MR, Donfack J, Choi SS, Conley SF, Derkay CS, Myer CM 3rd, Ehrlich GD, Post JC: Unique challenges of obtaining regulatory approval for a multicenter protocol to study the genetics of RRP and suggested remedies. Otolaryngol Head Neck Surg 2006;135:189-196.

24 Beskow LM, Burke W, Merz JF, Barr PA, Terry S, Penchaszadeh VB, Gostin LO, Gwinn $\mathrm{M}$, Khoury MJ: Informed consent for population-based research involving genetics. JAMA 2001;286:2315-2321.

25 National Human Genome Research Institute (NHGRI): Informed consent for genomics research. 2010. http://www.genome. gov/27026588. 